\title{
Online adaptive semi-active vibration damping of slender structures subject to moving loads
}

\author{
Dominik Pisarski ${ }^{1}$, and Andrzej Myśliński ${ }^{2, \star}$ \\ ${ }^{1}$ Institute of Fundamental Technological Research of Polish Academy of Sciences, 02-106 Warsaw, Poland \\ ${ }^{2}$ Systems Research Institute of Polish Academy of Sciences, 01-447 Warsaw, Poland
}

\begin{abstract}
The paper deals with the adaptive optimal semi-active control of the slender vibrating structures subjected to the moving loads. The deflection of the structure is governed by Euler-Bernoulli beam equation approximated by the system of bilinear ordinary differential equations. The damping function of the structure support is chosen as the control function. The optimal control problem consists in finding such bang-bang control function to minimize the energy as well as the vibrations of the carrying structure. Although the switched optimal control is a very efficient tool in the reduction of structure vibrations it is very sensitive with respect to changes of the speed of the traveling load. This paper deals with the development of the adaptive descent type algorithm that enables the update of the optimal controls in real time based on the measured speed of the traveling load or structure's state. The proposed algorithm uses reference optimal controls computed for the constant speeds and the set of functions describing the sensitivity of the system dynamics with respect to the measured parameters. Numerical computations are carried out for different speed scenarios of the moving load. The obtained numerical results indicate that the proposed adaptive controller can significantly outperform the reference optimal solutions.
\end{abstract}

\section{Introduction}

The high speed traveling loads interacting with the slender structures generate their vibrations and/or reduce their stability. The primary aim of the structural control is to reduce these undesired oscillations and to enhance the structure's stability [1]. Many control methods of vibrating structures, including adaptive controllers (see references in [2]) are proposed in literature. Most of the existing optimal adaptive controllers (see [3]) are based on complex iterative procedures that often do not guarantee convergence to a solution in the required time.

The aim of this paper is to develop and test new method that enables online adaptation of the optimal damping of the structure's supports according to both the measured speed of the traveling load and the estimated state of the structure. The control aim consists in finding the bangbang control function minimizing the objective functional related to the total energy of the structure. The design of the adaptive controller will be based on two optimal control subproblems. The first one will be the bilinear optimal control subproblem involving some given reference passage velocity. For this subproblem, we will formulate the necessary optimality conditions and identify the switched structure of the optimal controls. For the assumed reference velocity, the set of optimal switching times will be computed off line by solving the corresponding two point boundary value problem. In the second subproblem, intended for the sequential solution, we will consider the actual measured velocity. To find the set of optimal

\footnotetext{
^Corresponding author: myslinsk@ibspan.waw.pl
}

switching times for this subproblem, we will use the simple gradient-based iterative procedure involving the optimal solutions computed for the reference speed and the set of functions describing the sensitivity of the system dynamics with respect to the measured velocity. The sensitivity of reference solutions with respect to velocity is calculated based on Taylor series expansion rather than on the analysis of the second order optimality conditions as in $[2,4]$. Since the sensitivity functions can be computed off line the solution to the second subproblem can be calculated very quickly. This feature allows the frequent update of the optimal controls. The performance of the designed adaptive method will be validated by means of numerical simulations for different speed scenarios of the moving load.

\section{Structure model}

Consider the carrying structure supported by the set of controlled dampers as illustrated in Fig. 1. The structure is governed by one-dimensional fourth order EulerBernoulli beam equation [5] and is characterized by the bending stiffness $E I$ and density per unit length $\mu$. The interaction between the mass and the beam is represented by contact point. The inertial forces associated to the mass are neglected [5]. The position and the velocity of the moving mass at time $t$ are denoted by $\xi_{M}(t)$ and $v(t)$, respectively. The mass position is computed by formula $\xi_{M}(t)=\int_{0}^{t} v(\bar{t}) \mathrm{d} \bar{t}$. 


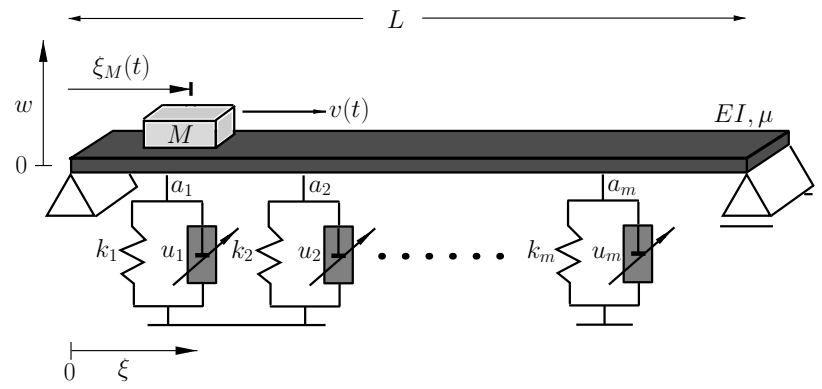

Fig. 1. A span supported by the set of controlled dampers and subjected to the traveling mass.

\subsection{ODE representation}

Using the separation technique the transverse deflection $w(\xi, t)$ of the vibrating beam at point $\xi$ and time $t$ governed by Euler-Bernoulli equation can be approximated [5] by the following Fourier series:

$$
w(\xi, t)=\frac{2}{L} \sum_{j=1}^{\infty} V_{j}(t) \theta_{j}(\xi), \quad \theta_{j}(\xi)=\sin (\bar{j} \pi \xi / L) .
$$

We will rely on the approximated solutions by taking $\bar{j}$ from 1 to $n=10$. The state vector $x(t) \in R^{2 n}$ is defined as

$$
x(t)=\left[V_{1}(t), \dot{V}_{1}(t), V_{2}(t), \dot{V}_{2}(t), \ldots, V_{n}(t), \dot{V}_{n}(t)\right]^{T},
$$

where $\dot{V}_{j}=d V_{j} / d t, j=1, \ldots, n$. Let us also introduce the system matrices $A_{2 n \times 2 n}, B_{2 n \times 2 n}^{i}, i=1,2, . ., m$ equal to: $(A)_{j k}=1$ for $(j, k)=(2 l-1,2 \bar{l}), \bar{l}=1,2, \ldots, n$, $(A)_{j k}=-\frac{E I}{\mu} \frac{(j / 2)^{4} \pi^{4}}{L^{4}}$ for $(j, k)=(2 \bar{l}, 2 \bar{l}-1), \bar{l}=1,2, . ., n$, $(A)_{j k}=-\frac{2}{\mu L} \sum_{i=1}^{m} \sin \frac{((j+1) / 2) \pi a_{i}}{L} \sin \frac{(k / 2) \pi a_{i}}{L}$ for $(j, k)=(2 \bar{l}-$ $1,2 \hat{l}) \bar{l}, \hat{l}=1,2, \ldots, n,(A)_{j k}=0$ in other cases. Moreover for $i=1, \ldots, m,\left(B^{i}\right)_{j k}=-\frac{2}{\mu L} \sin \frac{(j / 2) \pi a_{i}}{L} \sin \frac{(k / 2) \pi a_{i}}{L}$ for $(j, k)=(2 \bar{l}, 2 \hat{l}) \bar{l}, \hat{l}=1,2, \ldots, n$ and $\left(B^{i}\right)_{j k}=0$ else. The excitation vector $F_{2 n \times 1}$ is given by: $(F)_{j}=-\frac{M g}{\mu} \sin \frac{(j / 2) \pi v t}{L}$ for $j=2 \bar{l}, \bar{l}=1,2, \ldots, n$ and $(F)_{j}=0$ else. The beam dynamic equation can be written $[2,5]$ as the following system of the ordinary differential equations in time interval $t \in[0, T]$ with the fixed final time $T>0$ :

$$
\begin{gathered}
\dot{x}(t)=A x(t)+\sum_{i=1}^{m} u_{i}(t) B^{i} x(t)+F(v, t), \\
x(0)=x_{0} .
\end{gathered}
$$

The time horizon $T$ is equal to the time of the beam passage, i.e., $T=L / v$. For $j=1,2, \ldots, n$ the function $x_{0} \in R^{2 n}$ in the initial condition is given and equal to

$$
x_{0}=\left[V_{1}(0), \dot{V}_{1}(0), V_{2}(0), \dot{V}_{2}(0), \ldots, V_{n}(0), \dot{V}_{n}(0)\right]^{T} .
$$

Elements $V_{j}^{-}(0)$ and $\dot{V}_{j}^{-}(0)$ are computed based on the original boundary conditions imposed on vibrating beam [2]. The function $x_{0} \in R^{2 n}$ is assumed to be bounded. The matrices $A$ and $B^{i}, i=1, \ldots, m$, are bounded. Each control input $u_{i}(t), i=1, \ldots, m, t \in[0, T]$ is assumed bounded by two positive real values $u_{i}^{\min }$ and $u_{i}^{\max }$ corresponding to the minimum and maximum values of the admissible damping coefficients, i.e., $u=u(t) \stackrel{\text { def }}{=}\left\{u_{i}(t)\right\}_{i=1}^{m}, u \in \mathcal{U} \stackrel{\text { def }}{=}$ $\left[u_{i}^{\text {min }}, u_{i}^{\text {max }}\right]^{m} \subset \mathbb{R}_{+}^{m}$. Moreover $0<u_{i}^{\text {min }}<u_{i}^{\max } \leq \tilde{\alpha}$, where for $i=1, \ldots, m \tilde{\alpha}>u_{i}^{m i n}$ is a given real constant.

Note that the control variable $u$ appears linearly in the state system (3). Due to the middle term in the right hand side of (3), the system is classified as the bilinear [2].

The excitation vector $F=F(v, t)=\left\{F_{i}(v, t)\right\}_{i=1}^{2 n}$ is dependent on velocity $v=v(t):[0, T] \rightarrow R$. The velocity $v$ is assumed bounded, i.e., for all $t \in[0, T]$ $v^{\min }<v(t)<v^{\max }$ where $v^{\min }$ and $v^{\max }$ are a given real constants and continuously differentiable. Each component function $F_{i}(v, t): R \times[0, T] \rightarrow R, i=1, \ldots, 2 n$, of the excitation vector $F$ is assumed bounded and continuously differentiable with respect to time $t$ and velocity $v$. In the paper, the bang-bang type of control $u \in U$ is considered. It generates the discontinuity of the right hand side of the system (3).

Let $v_{\text {ref }} \in R$ denote a given reference velocity of the moving load. For such a velocity and a positive definite $2 n \times 2 n$ matrix $Q$ let us recall from [6-8] the optimal control problem considered for the system (3): For a given velocity $v_{\text {ref }}$ find the pair of functions $(u(t), x(t)) \in \mathcal{U} \times R^{2 n}$ minimizing the objective functional

$$
J(u)=J\left(x(u), v_{r e f}\right)=\int_{0}^{T} x^{T}(t) Q x(t) \mathrm{d} t
$$

subject to the constraints on the interval $[0, T]$

$$
\begin{gathered}
\dot{x}(t)=A x(t)+\sum_{i=1}^{m} u_{i}(t) B^{i} x(t)+F\left(v_{\text {ref }}, t\right), \\
x(0)=x_{0}^{v_{\text {ref }}} .
\end{gathered}
$$

The solution to problem (5)-(6) will be later referred to as $\left(u^{\star v_{\text {ref }}}(t), x^{\star v_{\text {ref }}}(t)\right)$. The objective functional $J$ : $R^{2 n} \times R \rightarrow R$ is associated with the energy of the structure. The aim of this optimal control problem is to find the control function, i.e., variable damping coefficients, so that for a given velocity, the energy of the beam subjected to the traveling load is minimized. As it is shown in [6], by minimizing the objective functional (5) we also provide the smooth trajectory of the moving load. The existence of an optimal solution $u^{\star v_{\text {ref }}}(t) \in U$ to problem (5)-(6) follows from Filippov theorem and the continuity of the objective functional (for details see [9]).

\subsection{Necessary optimality condition for problem (5)-(6)}

Let us introduce Hamiltonian function $H\left(t, x, u, p, v_{r e f}\right)$ : $[0, T] \times R^{2 n} \times \mathcal{U} \times R^{2 n} \times R \rightarrow R$ defined as follows:

$$
\begin{gathered}
H\left(t, x, u, p, v_{r e f}\right)=p^{T}(t)(A x(t)+ \\
\left.\sum_{i=1}^{m} u_{i}(t) B^{i} x(t)+F\left(v_{r e f}, t\right)\right)-x^{T}(t) Q x(t),
\end{gathered}
$$

where $p(t) \in R^{2 n}$ is a column vector denoting the adjoint state. The part of Hamiltonian function associated with the control function $u$ is called the switching function [4]

$$
\sigma\left(t, x, p, v_{r e f}\right)=\left(\sigma_{1}\left(t, x, p, v_{r e f}\right), \ldots\right.
$$




$$
\left.\sigma_{m}\left(t, x, p, v_{r e f}\right)\right)
$$

$\sigma_{i}\left(t, x, p, v_{r e f}\right)=p^{T}(t) B^{i} x(t)$ for $i=1,2, \ldots, m$. Therefore, the necessary optimality condition for (5)-(6) takes the following form $[2,5]$ : If $u^{\star v_{\text {ref }}}(t)$ is the optimal control for the problem (5)-(6) then there exists absolutely continuous function $p:[0, T] \rightarrow R^{2 n}$ satisfying the following conditions:

$$
\begin{array}{r}
\dot{p}(t)=-\frac{\partial H\left(t, x, u, p, v_{r e f}\right)}{\partial x}, \quad p(T)=0, \\
H\left(t, x, u^{\star v_{r e f}}, p, v_{r e f}\right)=\min _{u \in \mathcal{U}} H\left(t, x, u, p, v_{r e f}\right) .
\end{array}
$$

From the minimum condition (10) for the admissible control set $\mathcal{U}$ results the following control law for the $i$ th optimal control component

$$
u_{i}^{\star v_{\text {ref }}}(t)= \begin{cases}u_{i}^{\text {min }}, & \text { if } \sigma_{i}(t)<0, \\ u_{i}^{\text {max }}, & \text { if } \sigma_{i}(t)>0, \\ \text { undetermined, } & \text { if } \sigma_{i}(t)=0 .\end{cases}
$$

Throughout the rest of the paper, by the $k$ th switching time we will mean the column vector $\tau_{k}=\left(\tau_{k 1}, \tau_{k 2}, \ldots, \tau_{k m}\right)$ where $\tau_{k i}$ is associated with the control component $u_{i}$.

\section{Sensitivity of optimal bang-bang controls with respect to velocity}

Assume that for a given velocity $v_{\text {ref }}$ the optimal control structure $\tau_{k}$ for the problem (5)-(6) is known. As it was shown in [5], the solution to optimal control problem (5)(6) exhibits significant sensitivity with respect to changes in the speed $v$ of the traveling load. Denote by $t^{\prime} \in[0, T)$ the measurement time instant of the traveling load and the state of the structure. Assuming that for the chosen time instant $t^{\prime}$ the real speed $v=v\left(t^{\prime}\right)$ of the traveling load and the real state $x\left(t^{\prime}\right)$ of the structure are known from the measurements our goal is to develop a method that enables the real-time adaptation of the optimal control functions according to both measured values. The method will use the reference optimal control functions computed for a given reference speed and the set of functions describing the sensitivity of the system dynamics to the measured parameters. Assume that the velocity perturbation

$$
d v=v-v_{r e f},
$$

is suitably small. Let us now consider new finitedimensional optimization problem involving the perturbed switching times as the optimization variables. First, we will introduce the following assumptions [2, 4]:

A1 The optimal control $u^{\star v_{\text {ref }}}$ (as well as the switching function $\sigma(t)$ ) has a finite number of switching times (zeroes) $\tau_{k}^{\star v_{\text {ref }}}, k=1, \ldots, K$ satisfying

$$
0=\tau_{0}^{\star v_{\text {ref }}}<\tau_{1}^{\star v_{\text {ref }}}<\tau_{k}^{\star v_{\text {ref }}}<\ldots<\tau_{K+1}^{\star v_{\text {ref }}}=T .
$$

A2 There exists a set of twice continuously differentiable functions $u_{k}(t, x)$ such that the control $u^{\star v_{\text {ref }}}(t)$ is given in a state feedback form, i.e., for $k=1, \ldots, K+1$ it holds

$$
u^{\star v_{r e f}}(t)=u_{k}\left(t, x^{\star v_{r e f}}(t)\right), \quad \forall t \in\left(\tau_{k-1}^{\star v_{r e f}}, \tau_{k}^{\star v_{r e f}}\right) .
$$

A3 The time derivative $\dot{\sigma}$ of the switching function $\sigma$ is assumed not to vanish, i.e., the strict bang-bang property [4] is assumed to be satisfied

$$
\dot{\sigma}\left(\tau_{k}^{\star v_{r e f}}\right) \neq 0, \quad k=1, \ldots K
$$

Based on the assumptions $\mathbf{A 1}-\mathbf{A} \mathbf{3}$ we will reformulate $[2,4]$ the optimal control problem (5)-(6) as the finitedimensional nonlinear induced optimization problem in terms of the switching times rather than solve it directly as the original problem (5)-(6). Let us introduce the matrix of switching times

$$
\begin{gathered}
\eta=\left(\tau_{1}, \tau_{2}, \ldots, \tau_{K}\right) \in R^{K \times m}, \\
0=\tau_{0}<\tau_{1}<\tau_{k}<\ldots<\tau_{K+1}=T,
\end{gathered}
$$

taken from a neighborhood of the optimal reference vector $\eta^{\star v_{\text {ref }}}=\left(\tau_{1}^{\star v_{\text {ref }}}, \tau_{2}^{\star v_{\text {ref }}}, \ldots, \tau_{K}^{\star v_{\text {ref }}}\right)$. For any $\eta$ satisfying (17), we will write $u^{\eta}(t)$ for the nonsingular bang-bang control for $\tau_{k-1}<t<\tau_{k}, k=1, \ldots, K+1$ defined as follows:

$$
u^{\eta}(t)=\left(u_{1}^{\eta}(t), u_{2}^{\eta}(t), \ldots, u_{m}^{\eta}(t)\right)=\left(u_{k 1}, u_{k 2}, \ldots, u_{k m}\right) .
$$

Let us denote by $\mathcal{K}$ the following subset of the switching times indicators:

$$
\mathcal{K}=\left\{k: k=1, \ldots, K+1, \tau_{k} \geq t^{\prime}\right\} .
$$

For the control $u^{\eta}(t), \eta=\left(\left(\tau_{k}\right)_{k \in \mathcal{K}}\right)$, let $x=x\left(t, x\left(t^{\prime}\right), \eta, v\right)$ be the absolutely continuous function satisfying the initial value problem

$$
\begin{aligned}
& \dot{x}(t)=A x(t)+\sum_{i=1}^{m} u_{i}^{\eta}(t) B^{i} x(t)+F(v, t), \\
& \text { for } \tau_{k-1}<t<\tau_{k}, \quad k \in \mathcal{K}, \quad x\left(t^{\prime}\right)=x_{0}^{v} .
\end{aligned}
$$

Referring to problem (5)-(6), we have $x\left(t, x^{\star v_{r e f}}\left(t^{\prime}\right), \eta^{\star v_{r e f}}\right.$, $\left.v_{\text {ref }}\right)=x^{\star v_{\text {ref }}}(t)$. Using the solution of the state equation (20), we can now formulate the parametric optimization problem in terms of $\eta$ as the optimization variable: For a given velocity $v$ find $\eta=\left(\left(\tau_{k}\right)_{k \in \mathcal{K}}\right)$ minimizing the objective functional

$$
J(\eta)=J(x(\eta), v)=\int_{t^{\prime}}^{T} x^{T}(t) Q x(t) \mathrm{d} t,
$$

subject to the constraints $(20)$ on the interval $\left[t^{\prime}, T\right]$. The solution to problem (21) will be from now on referred to as $\eta^{\star v}=\left(\left(\tau_{k}^{\star v}\right)_{k \in \mathcal{K}}\right)$.

\subsection{Directional derivatives with respect to switching time}

To solve the optimization problem (21) with constraints (20) we develop an iterative algorithm based on the method of the steepest descent. For that purpose we need to estimate the relevant derivatives corresponding to the directions of the descent for the decision parameter $\eta$. It can be verified (see, for instance, [5]) that the derivative of the 
objective functional (21) with respect to the $k$ th switching time of the $i$ th control component is given by

$$
\frac{d J}{d \tau_{k i}}=-\int_{t^{\prime}}^{T}\left(\frac{\partial H}{\partial \tau_{k i}}\right)_{\mid \tau_{k i}} \mathrm{~d} t .
$$

From (11) it yields

$$
\begin{gathered}
\frac{d J}{d \tau_{k i}}=\left(u_{(k+1) i}-u_{k i}\right) p^{T}\left(\tau_{k i},\right. \\
\left.x\left(t^{\prime}\right),\left(\tau_{k}\right)_{k \in \mathcal{K}}, v\right) B^{i} x\left(\tau_{k i}, x\left(t^{\prime}\right),\left(\tau_{k}\right)_{k \in \mathcal{K}, v) .}\right.
\end{gathered}
$$

Here, $u_{k i}$ is the value of the $i$ th control component in the time interval $t \in\left(\tau_{k-1}, \tau_{k}\right), k \in \mathcal{K}$. In order to evaluate the derivatives (23), we employ approximations of the state $x$ and the adjoint state $p$. The approximation of the state $x$, denoted later by $x^{a p p}$, will combine the optimal reference state $x^{\star v_{\text {ref }}}$ and the appropriate differentials due to the changes in the speed of the moving load, the initial condition, and the switching times. Similarly, for the approximate adjoint state $p$, later referred to as $p^{a p p}$, we will use $p^{\star v_{\text {ref }}}$ and the relevant differentials.

It follows from the assumption (13) on the velocity perturbation that the perturbation of the initial state $d x=$ $x\left(t^{\prime}\right)-x^{\star r e f}\left(t^{\prime}\right)$ can also be assumed to be suitably small. It implies small perturbations of the switching times, defined as follows:

$$
\begin{gathered}
d \tau_{k}=\left(d \tau_{k 1}, d \tau_{k 2}, \ldots, d \tau_{k m}\right), \\
d \tau_{k i}=\tau_{k i}-\tau_{k i}^{\star r e f}, \quad i=1,2, \ldots, m, k \in \mathcal{K} .
\end{gathered}
$$

By using the perturbations (13) and (24) the state $x\left(t, x\left(t^{\prime}\right),\left(\tau_{k}\right)_{k \in \mathcal{K}}, v\right)$ can be evaluated as the function dependent on

$$
x\left(t, x^{\star r e f}\left(t^{\prime}\right)+d x,\left(\tau_{k}^{\star r e f}+d \tau_{k}\right)_{k \in \mathcal{K}}, v_{r e f}+d v\right) .
$$

Since for $t \in\left[t^{\prime}, T\right]$ we have $x^{\star v_{\text {ref }}}(t)=x\left(t, x^{\star r e f}\left(t^{\prime}\right)\right.$, $\left.\left(\tau_{k}^{\star r e f}\right)_{k \in \mathcal{K}}, v_{\text {ref }}\right)$, by using Taylor series expansion formula and the standard arguments [2] we can employ the following approximation of the state function:

$$
\begin{gathered}
x^{a p p}(t)=x^{\star v_{\text {ref }}}(t)+\left(\frac{\partial x}{\partial v}\right)_{\mid t} d v+\frac{1}{2}\left(\frac{\partial^{2} x}{\partial v^{2}}\right)_{\mid t} d v^{2}+ \\
\left(\frac{\partial x}{\partial x_{0}}\right)_{\mid t} d x+\sum_{i=1}^{m} \sum_{k \in \mathcal{K}}\left(\frac{\partial x}{\partial \tau_{k i}}\right)_{\mid t} d \tau_{k i} .
\end{gathered}
$$

For $i=1,2, \ldots, m$ and $k \in \mathcal{K}$ let us introduce the following definitions of the sensitivity functions

$$
S_{v}^{x}=\frac{\partial x}{\partial v}, \quad S_{v v}^{x}=\frac{\partial^{2} x}{\partial v^{2}}, \quad S_{x_{0}}^{x}=\frac{\partial x}{\partial x_{0}}, \quad S_{\tau_{k i}}^{x}=\frac{\partial x}{\partial \tau_{k i}} .
$$

Denote by $f$ the right hand side of equation (6) for $t \in$ $\left[t^{\prime}, T\right]$. Thus, $f=\dot{x}$ and due to (20) we have the following relations:

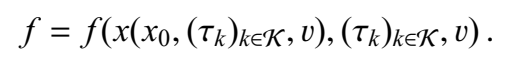

Thus, using the standard sensitivity approach [9] and differentiation with respect to the perturbed parameters we obtain ordinary differential equations satisfied by the sensitivity functions (27) in $\left[t^{\prime}, T\right]$ :

$$
\begin{gathered}
\dot{S}_{v}^{x}=\frac{\partial f}{\partial x} S_{v}^{x}+\frac{\partial f}{\partial v}, \quad S_{v}^{x}\left(t^{\prime}\right)=0, \\
\dot{S}_{v v}^{x}=\frac{\partial f}{\partial x} S_{v v}^{x}+\frac{\partial^{2} f}{\partial v^{2}}, \quad S_{v v}^{x}\left(t^{\prime}\right)=0, \\
\dot{S}_{x_{0}}^{x}=\frac{\partial f}{\partial x} S_{x_{0}}^{x}, \quad S_{x_{0}}^{x}\left(t^{\prime}\right)=1,
\end{gathered}
$$

and for $i=1,2, \ldots, m$ and $k \in \mathcal{K}$

$$
\dot{S}_{\tau_{k i}}^{x}=\frac{\partial f}{\partial x} S_{\tau_{k i}}^{x}+\frac{\partial f}{\partial \tau_{k i}}, \quad S_{\tau_{k i}}^{x}\left(t^{\prime}\right)=0
$$

From (6) we obtain

$$
\begin{gathered}
\frac{\partial f}{\partial x}=A+\sum_{i=1}^{m} u_{i} B^{i}, \\
\frac{\partial f}{\partial \tau_{k i}}=\left(u_{(k+1) i}-u_{k i}\right) B^{i} x \delta\left(t-\tau_{k i}\right),
\end{gathered}
$$

where $\frac{\partial f}{\partial v}=\frac{\partial F}{\partial v}, \frac{\partial^{2} f}{\partial v^{2}}=\frac{\partial^{2} F}{\partial v^{2}}$. The approximation $p^{a p p}$ of the adjoint state $p$ is based on the adjoint state $p^{\star v_{\text {ref }}}$ corresponding to the optimal state $x^{\star v_{\text {ref }}}$. It is computed similarly as the approximation $x^{a p p}$ of the state function $x$, i.e., Taylor series expansion (26) is used with the adjoint function $p$ rather than the state function $x$.

\section{Optimal control algorithm}

The developed adaptive control numerical algorithm is based on the concept of the Receding Horizon Control. This class of algorithms employs the solutions to the finite horizon optimal control problems where both the initial and the final times are repetitively being pushed forward. In this paper, a sequence of problems (21) with incrementally increasing time $t^{\prime}$, but fixed final time $T$, is considered. The final time is equal to the time in which a moving load is passing the structure with the constant velocity $v_{r e f}$. The fixed final time enables us to employ the optimal reference solutions. Based on the measurements of the actual velocity $v\left(t^{\prime}\right)$ and the actual state $x\left(t^{\prime}\right)$ as well as using the formulas for the objective functional derivatives (23) combined with the formula (26) to calculate the approximation of the state and adjoint functions the algorithm is able to compute the descent directions to update the set of the optimally switched controls. Remark that all of the sensitivity state and adjoint functions (29)-(32) can be precomputed off line and stored in a controller's memory. Since the computation of the values of the objective functional derivatives (23) can be executed instantaneously, the adaptive method can be implemented in real-time. For detailed description of the algorithm see [2].

\section{Numerical examples}

The optimal control problem (21) with constraints (20) has been solved numerically for several passage scenarios including both constant and varying speed profiles. For each 
scenario the same reference optimal controls computed for given constant reference speed is used. Our primary goal is to estimate the range of velocity perturbation that guarantees high performance of the designed method. Matrix $Q$ in the objective functional (21) is assumed as the identity matrix and the time horizon $T$ is computed for $L=4[m]$. Here $v(t)$ is the actual velocity in the speed scenario. Other parameters are set as equal to $n=10, m=4$, $E I=801\left[\mathrm{Nm}^{2}\right], \mu=2.3[\mathrm{~kg} / \mathrm{m}], u^{\min }=500[\mathrm{~N} / \mathrm{m}]$, $u^{\max }=5000[\mathrm{~N} / \mathrm{m}], a_{i}=0.2 \mathrm{~L}, 0.4 \mathrm{~L}, 0.6 \mathrm{~L}, 0.8 \mathrm{~L}, \mathrm{M}=$ $4[\mathrm{~kg}]$. In the simulations, maximal number of iterations is set to $l_{\max }=20$ and stop criterion $\epsilon=3 \cdot 10^{-10}$. Four control functions $u_{i}, i=1,2,3,4$, associated with four controlled dampers are used. For each control function $u_{i}$ two switching times $\tau_{1 i}$ and $\tau_{2 i}$ are assumed (see Fig. 2). The structure of the controls is defined as follows:

$$
u_{i}(t)= \begin{cases}u^{\text {min }}, & \text { if } \quad 0 \leq t<\tau_{1 i}, \quad i=1,2,3,4 \\ u^{\text {max }}, & \text { if } \tau_{1 i} \leq t \leq \tau_{2 i}, \quad i=1,2,3,4 \\ u^{\text {min }}, & \text { if } \tau_{2 i}<t \leq T, \quad i=1,2,3,4\end{cases}
$$

The solution to the reference optimal control problem (5)-(6) has been computed for the moving load speed $v_{\text {ref }}=8[\mathrm{~m} / \mathrm{s}]$ and zero initial condition $x_{0}^{v_{\text {ref }}}=0$. The corresponding time horizon is equal to $T=0.5[s]$. The time interval $[0, T]$ is represented by the following set of time instants $\left\{t_{j}\right\}_{j=0,1, \ldots, 1000,} t_{0}=0, t_{1000}=T$. For this representation we obtain the set of optimal switching times for problem (5)- (6):

$$
\begin{array}{lll}
\text { damper no. } 1 & \tau_{11}^{\star v_{\text {ref }}}=t_{1}, & \tau_{21}^{\star v_{\text {ref }}}=t_{366}, \\
\text { damper no. } 2 & \tau_{12}^{\star v_{\text {ref }}}=t_{152}, & \tau_{22}^{\star v_{\text {ref }}}=t_{596}, \\
\text { damper no. } 3 & \tau_{13}^{\star v_{\text {ref }}}=t_{322}, & \tau_{23}^{\star v_{\text {ref }}}=t_{732}, \\
\text { damper no. } 4 & \tau_{14}^{\star v_{\text {ref }}}=t_{80}, & \tau_{24}^{\star v_{\text {ref }}}=t_{972} .
\end{array}
$$

The results for the exact optimal controls, later referred to as the optimal controls and denoted by $u^{\star \star}$ are computed as the solutions to the problem (5)-(6), where instead of the reference velocity $v_{\text {ref }}$ and the initial state $x_{0}^{v_{\text {ref }}}$ we assume the actual speed profiles as equal either to constant velocity $v_{\text {const }}$ or piecewise linear variable velocity $v_{v a r}$. For each case, the optimal control is assumed to preserve two switches structure as given by (35).

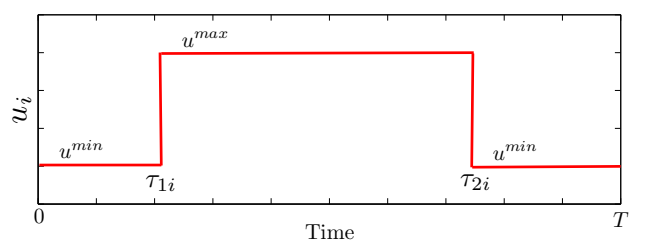

Fig. 2. The assumed structure of the switched controls $u_{i}, i=$ $1,2,3,4$.

The first set of simulations has been carried out for perturbed velocity scenarios. We have considered three scenarios of constant speed $v_{\text {const }}$ and three scenarios of varying speed $v_{v a r}$. The assumed profile of the varying speed $v_{v a r}$ is displayed in Fig. 3. For each of the constant speed

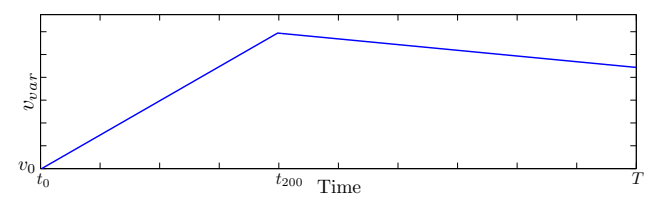

Fig. 3. The profile of the varying speed $v_{v a r}$ used in the simulations.

Table 1. The values of the objective function $J$ in the cases of the perturbed velocity scenarios.

\begin{tabular}{|c|c|c|c|c|}
\hline $\begin{array}{c}\text { Speed } \\
\text { variant } \\
\text { in }[\mathrm{m} / \mathrm{s}]\end{array}$ & $\begin{array}{c}\text { Passive } \\
\text { case } \\
\left(u=u_{\text {max }}\right)\end{array}$ & $\begin{array}{c}\text { Reference } \\
\text { optimal } \\
\text { control } \\
\left(u=u^{\star v_{\text {ref }}}\right)\end{array}$ & $\begin{array}{c}\text { Adaptive } \\
\text { control } \\
\left(u=u^{\star v}\right)\end{array}$ & $\begin{array}{c}\text { Optimal } \\
\text { control } \\
\left(u=u^{\star \star}\right)\end{array}$ \\
\hline \hline$v_{\text {const }}=8$ & 1.0000 & 0.4801 & 0.4801 & 0.4801 \\
\hline$v_{\text {const }}=9$ & 1.0000 & 0.5295 & 0.4990 & 0.4881 \\
\hline$v_{\text {const }}=12$ & 1.0000 & 0.9081 & 0.5448 & 0.5327 \\
\hline$v_{\text {var }}, v_{0}=8$ & 1.0000 & 0.5136 & 0.4939 & 0.4855 \\
\hline$v_{\text {var }}, v_{0}=9$ & 1.0000 & 0.5859 & 0.5202 & 0.5025 \\
\hline$v_{\text {var }}, v_{0}=12$ & 1.0000 & 1.1314 & 0.5696 & 0.5463 \\
\hline
\end{tabular}

scenarios $v_{\text {const }}$, we assume that the traveling velocity is within the range of $8-12[\mathrm{~m} / \mathrm{s}]$. The same range is assumed for the starting velocity $v_{0}$ in the cases of the varying speed $v_{\text {var }}$.

The comparison of the values of the objective function for the set of considered speed variants is presented in Table 1. For each speed scenario the objective values for the optimal, the reference and the adaptive control are normalized to the passive case. For moving load velocities higher than $8[\mathrm{~m} / \mathrm{s}]$, the adaptive strategy outperforms significantly (by $40.1 \%$ or $43.1 \%$ for velocity $12[\mathrm{~m} / \mathrm{s}]$ ) the system driven by the open-loop reference controls. On the other hand this strategy results in only $2.2 \%$ or $4.2 \%$ loss compared to the optimal case.

Fig. 4 and 5 display the evolution of the adaptive switching times during the moving load passage with velocity $v_{\text {const }}=12[\mathrm{~m} / \mathrm{s}]$. For the initial values, we assumed the set of the corresponding reference optimal solutions. Then, at each time instant, the switching times were repetitively updated until the time $t_{667}$ corresponding to the end of the passage. The largest change in switching times values was observed at the beginning of the adaptive process. The convergence of the algorithm's gradient-based pro-

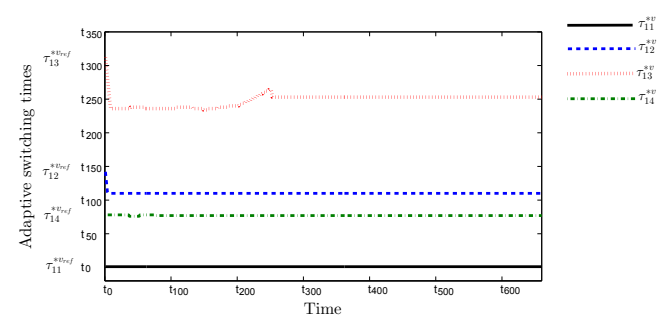

Fig. 4. Evolution of the optimal adaptive switching times $\left\{\tau_{1 i}^{\star v}\right\}$ during the moving load passage for the case $v_{\text {const }}=12[\mathrm{~m} / \mathrm{s}]$. The set of optimal reference switching times $\left\{\tau_{1 i}^{\star v_{r e f}}\right\}$ is assumed for the initial values. 


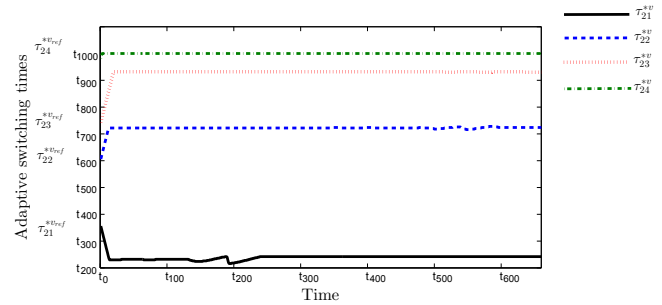

Fig. 5. Evolution of the optimal adaptive switching times $\left\{\tau_{2 i}^{\star v}\right\}$ during the moving load passage for the case $v_{\text {const }}=12[\mathrm{~m} / \mathrm{s}]$. The set of optimal reference switching times $\left\{\tau_{2 i}^{\star v_{r e f}}\right\}$ is assumed for the initial values.

cedure for different times instants is shown in Fig. 6. for the moving load passage with velocity $v_{\text {const }}=12[\mathrm{~m} / \mathrm{s}]$.

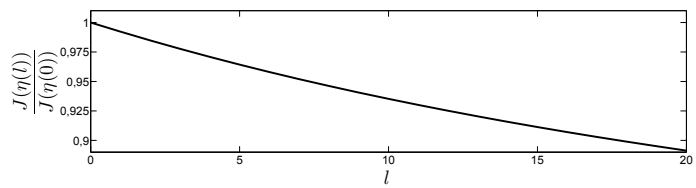

Fig. 6. Evolution of the objective functional during the iteration of the gradient-based procedure for the time instant $t_{0}$. The procedure is terminated by the condition $l=l_{\max }$.

In Fig. 7 and 8 we demonstrate the comparison of the adaptive, reference and optimal control signals as well as the trajectories of the moving load for $v_{\text {const }}=12[\mathrm{~m} / \mathrm{s}]$. One can notice that the switching times of the adaptive controls tend to approach their corresponding optimal control values.

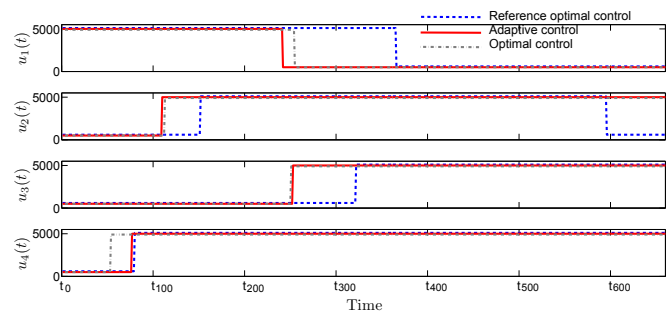

Fig. 7. Reference, adaptive, optimal controls. $v_{\text {const }}=12[\mathrm{~m} / \mathrm{s}]$.

\section{Conclusion}

Real time adaptive control method for structures subjected to a moving loads has been proposed and tested. The ob- tained results indicate that the proposed method can outperform the reference optimal solutions. Further numerical as well as experimental tests are being performed.

\section{References}

[1] C. Bajer, B. Dyniewicz, Arch. Appl. Mech. 79, 499508 (2009)

[2] D. Pisarski, A. Myśliński, Optim. Control Appl. Methods, Advance online publication, DOI:10.1002/oca.2321 (2017)

[3] M. Diehl, H.J. Ferreau, M. Abu-Khalaf, N. Haverbeke, Efficient numerical methods for nonlinear MPC and moving horizon estimation in nonlinear Model Predictive Control: Towards new challenging applications (Springer-Verlag, Berlin Heidelberg, 2009)

[4] G. Vossen, J. Optim. Theory Appl. 144, 409-429 (2010)

[5] D. Pisarski, Semi-active control system for trajectory optimization of a moving load on an elastic continuum (PhD Thesis, Institute of Fundamental Technological Research of Polish Academy of Sciences, Warsaw, 2012)

[6] D. Pisarski, J. Vibr. Control, Advance online publication, DOI:10.1177/1077546316657244 (2016)

[7] D. Pisarski, Math. Problems Eng. 2015, 1-12 (2015)

[8] D. Pisarski, C. Bajer, J. Sound Vibr. 329, 140-149 (2010)

[9] D. Liberzon, Calculus of variations and optimal control theory: A concise introduction (Princeton University Press, New Jersey, 2012)

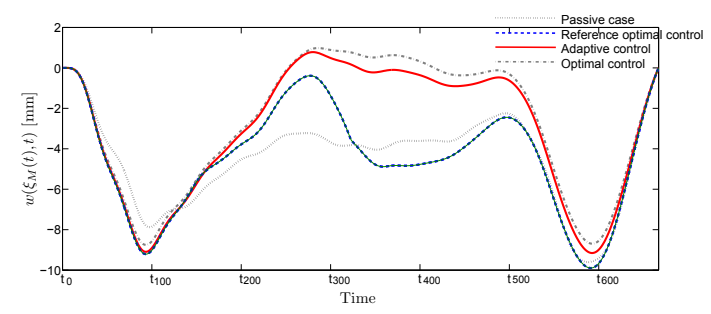

Fig. 8. Trajectories of the moving load for $v_{\text {const }}=12[\mathrm{~m} / \mathrm{s}]$. 